\title{
Idiopathic perforation of acalculous gallbladder after insertion of a transpapillary pancreatic stent
}

Authors

Institutions
Tomoko Katagiri' ${ }^{1}$, Atsushi Irisawa² ${ }^{2}$, Hiroto Wakabayashi' ${ }^{1}$, Takuya Tsunoda ${ }^{1}$, Hiroyuki Tomoda ${ }^{1}$, Ryo Saito $^{3}$, Shunji Kinuta ${ }^{3}$

${ }^{1}$ Department of Gastroenterology, Takeda general hospital, Aizuwakamatsu, Japan

${ }^{2}$ Department of Gastroenterology, Fukushima Medical University Aizu Medical Center, Aizuwamkamatsu, Japan

${ }^{3}$ Department of Surgery, Takeda general hospital. Aizuwakamatsu, Japan submitted 25. February 2016 accepted after revision 17. May 2016

\section{Bibliography}

Dol http://dx.doi.org/

10.1055/s-0042-109598

Published online: 10.8.2016

Endoscopy International Open 2016; 04: E838-E840

(c) Georg Thieme Verlag KG

Stuttgart · New York

E-ISSN 2196-9736

\section{Corresponding author}

\section{Atsushi Irisawa, MD, PhD}

Department of

Gastroenterology

Fukushima Medical University,

Aizu Medical Center

21-2, Maeda, Tanisawa,

Kawahigashi, Aizuwamkamatsu, 969-3492

Japan

irisawa@fmu.ac.jp
Background and study aims: Endoscopic retrograde pancreatocholangiography (ERCP) is associated with many types of adverse events (AEs) but idiopathic perforation of the gallbladder (IPGB) is very rare. Pancreatobiliary reflux is one of the factors involved with occurrence of IPGB [1]. Here we present a case of acalculous gallblad-

\section{Case report}

A 75-year-old man was admitted for treatment of acute alcoholic hepatitis. With enhanced computed tomography (CT), a pancreatic cystic lesion was observed incidentally. Endoscopic ultrasound (EUS) showed a multiple-cystic lesion with mural nodule in the tail of the pancreas and a diameter of $17 \mathrm{~mm}$. To obtain pancreatic juice for cytopathologic examination, ERCP was performed. Cannulation of the pancreatic duct was difficult and took more than 30 minutes (no cannulation into the bile duct during the procedure). We did not perform intraductal ultrasound and brushing because the cystic lesion was located on the end of the pancreas close to the splenic hilum.

Subsequently, an indwelling 5-Fr ENPD-tube was inserted in the pancreatic duct ( $\bullet$ Fig. 1). The ENPD tube had 4 side holes within $35 \mathrm{~mm}$ of its distal end. These holes were located in the pancreatic duct, not in the common channel. There was no obvious finding to suggest the presence of pancreaticobiliary maljunction based on magnetic resonance cholangiopancreatography (MRCP) prior to ERCP ( $\bullet$ Fig. 2). However, backflow of a small amount of contrast medium into the bile duct was present when a generous amount of contrast medium was injected into the pancreatic duct ( $\bullet$ Fig. 1, arrow). The occurrence of pancreatobiliary reflux without pancreaticobiliary maljunction was suspected. No AE was noted during the procedure. der perforation as an $\mathrm{AE}$ following the insertion of an indwelling endoscopic nasal pancreatic drainage (ENPD) tube (a pancreatic stent) to obtain pancreatic fluid. In this case, acute pancreatobiliary reflux might have been caused by the insertion of the ENPD-tube.

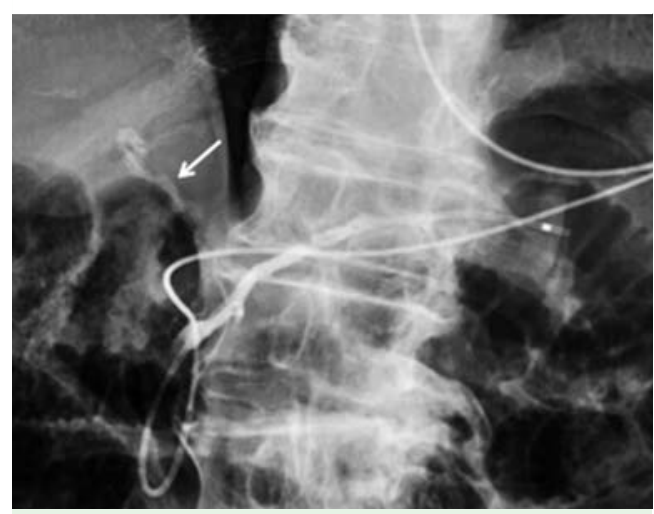

Fig. 1 A 5-Fr endoscopic nasopancreatic drainage tube indwelling in the pancreatic duct. There was no obvious finding suggestive of the presence of pancreaticobiliary maljunction, but a small amount of contrast medium flew back into the bile duct (arrow).

The patient complained of severe abdominal pain 11 hours after ERCP. Although about $200 \mathrm{~mL}$ of pancreatic fluid had been drained and the ENPD tube was removed 22 hours after the ERCP, the level of pain kept increasing. Contrast-enhanced CT showed a swollen gallbladder with an apparent thickening of the wall without evidence of biliary tract dilatation. In addition, massive ascites that had not been seen before ERCP were identified ( $\bullet$ Fig.3). Ascitic fluid was orange-colored and cloudy, and bilirubin and amylase concentrations $(38.9 \mathrm{mg} / \mathrm{dL}$ and $4325 \mathrm{IU} / \mathrm{l}$, normal limit is $1.0 \mathrm{mg} / \mathrm{dL}$ and $125 \mathrm{IU} / \mathrm{l}$ ) far exceeded the corresponding values measured in the blood on the same day $(0.6 \mathrm{mg} / \mathrm{dL}, 119 \mathrm{IU} / \mathrm{l})$. Perforation of the

\section{()(1) $\Theta \circledast$}




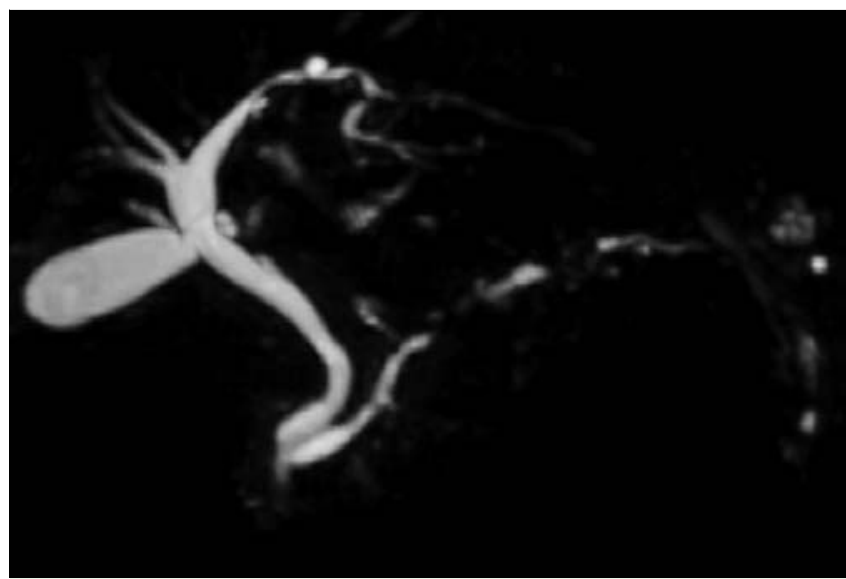

Fig.2 On magnetic resonance cholangiopancreatography, there were no obvious findings suggestive of the presence of pancreaticobiliary maljunction.

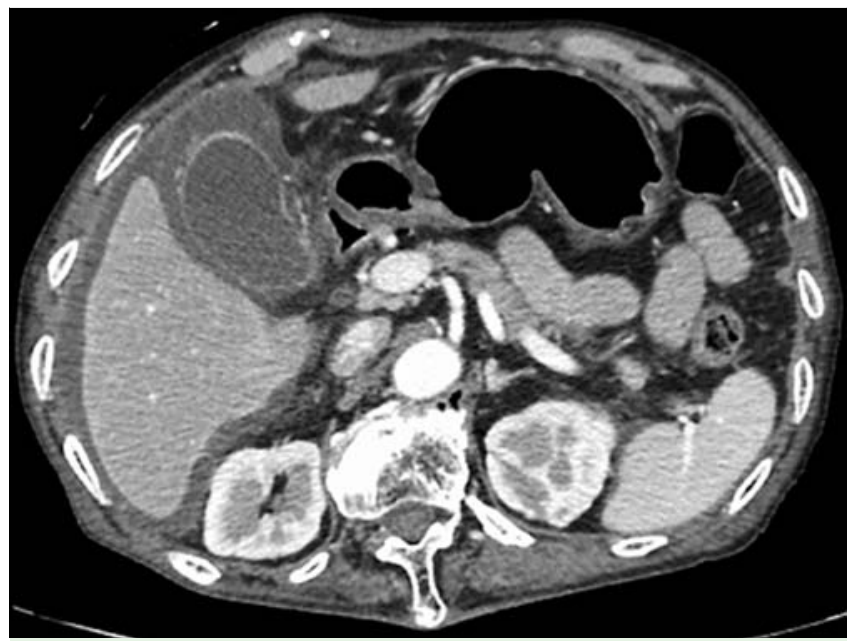

Fig. 3 Contrast-enhanced computed tomography (CT) at 30 hours postendoscopic retrograde cholangiopancreatography. CT showed a swollen gallbladder with apparent thickening of the wall and massive ascites.

duodenum but not the biliary tree was initially suspected, as both amylase and bilirubin levels in the ascites were high.

Subsequently, exploratory laparotomy was performed. Despite substantial bile in the peritoneal cavity, there was no evidence of duodenal perforation. The contents of the gallbladder were aspirated and its bilirubin and amylase levels were determined. Total bilirubin and amylase levels $(98.3 \mathrm{mg} / \mathrm{dL}, 1632 \mathrm{IU} / \mathrm{l})$ in the gallbladder were elevated as highly as the ascites. Therefore, acute cholecystitis with perforation due to acute pancreatobiliary reflux was diagnosed, and cholecystectomy was performed. Pathologic examination revealed that inflammatory cells had infiltrated throughout all layers, and abscess formation and necrosis with perforation were found in the subserosal layer ( $\bullet$ Fig.4). The structure of the mucosal epithelium was retained. No finding, such as hyperplastic change of gallbladder mucosa, was made that was suggestive of pancreaticobiliary maljunction and there were no gallbladder stones or tumor. The postoperative course was uneventful and the patient was discharged 9 days after surgery.

\section{Discussion}

\section{$\nabla$}

IPGB is rare and the underlying mechanisms are still unknown. Namikawa et al. [2] reported that thrombosis in the intramural vessels appeared to be related to the events leading to IPGB. In contrast, a few reports have suggested that pancreatobiliary maljunction may contribute to its etiology $[1,3]$. Nevertheless, it has been thought that pancreatobiliary reflux could result in several of various pathologic conditions in the biliary system, including acute cholecystitis, and pancreaticobiliary maljunction was not a necessary prerequisite [4].

Acute cholecystitis generally is well known as a cause of IPGB [5]. In our case, ultrasound and $\mathrm{CT}$ presented typical images indicative of acute cholecystitis. However, there was no stone or tumor that could cause the cholecystitis in the biliary tree. In this case, acute cholecystitis and perforation occurred after insertion of the indwelling ENPD-tube, thus it is believed that this pathologic condition occurred as an AE related to the ERCP and insertion of the indwelling ENPD-tube.

The cause of the gallbladder perforation was considered in our case. Pancreatobiliary reflux was highly implicated in the IPGB based on the high amylase concentration in the gallbladder contents [3]. However, in our case, pancreatobiliary maljunction was not identified on MRCP or ERCP. Past literature suggests that pancreatobiliary reflux can occur in individuals without pancreaticobiliary maljunction [6-8]. In particular, pancreatobiliary reflux has been frequently observed in individuals with a relatively long common channel [9], and in our case, it was observed on ERCP images. In fact, a small amount of contrast medium injected into the pancreatic duct back flowed into the bile duct, although MRCP did not indicate any pancreatobiliary maljunction. Thus, it was speculated that pancreatic fluid could easily flow into the bile duct due to the indwelling ENPD tube, which would eliminate space between the tube and the common channel. Consequently, the concentration of pancreatic enzymes in the biliary
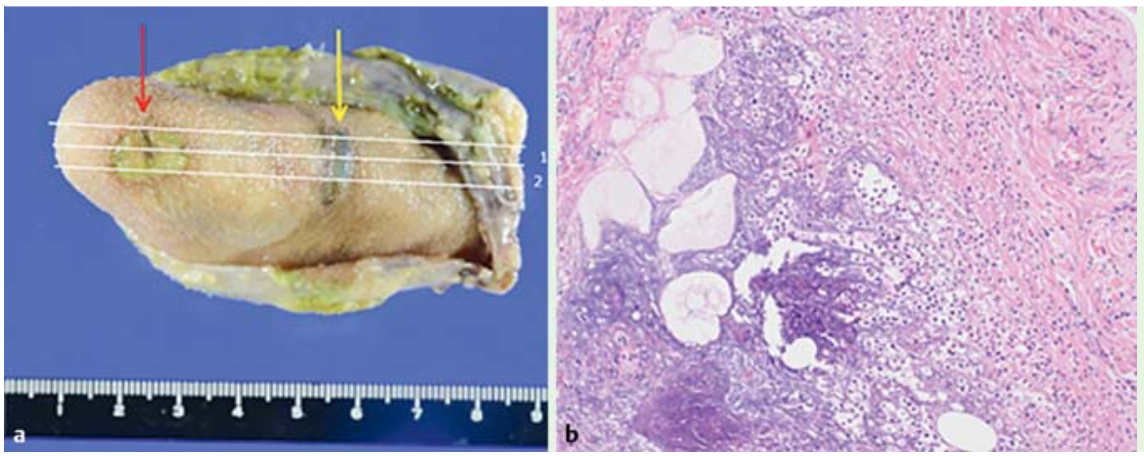

Fig. 4 a Resected Gallbladder. The red arrow is the site of a puncture made by the surgeon to aspirate the contents of the gallbladder. The yellow arrow indicates a perforation. b Microscopic examination at the site of perforation showed invasion of inflammatory cells throughout all layers and abscess formation and necrosis in the subserosal layer. 
tree rapidly increased and pooled in the gallbladder after insertion of the indwelling ENPD tube, potentially causing acute cholecystitis, and subsequently gallbladder perforation.

IPGB is rare as an AE associated with an indwelling ENPD tube. Although ENPD insertion is common in Japan, it is hard to explain why IPGB has not been observed in many other cases. Our patient might have a tendency to easily develop pancreatobiliary reflux because of the anatomy of the long common channel.

In conclusion, our case supports the suggestion that pancreatobiliary reflux is an important cause of IPGB. In addition, IPGB also should be recognized as a possible $\mathrm{AE}$ of pancreatic stent insertion, especially in patients who have a long common channel.

\section{Competing interests: None}

\section{References}

1 Hasegawa T, Udatsu $Y$, Kamiyama $M$ et al. Does pancreatico-biliary maljunction play a role in spontaneous perforation of the bile duct in children? Pediatr Surg Int 2000; 16: 550-553

2 Namikawa T, Kobayashi M, Okabayashi $T$ et al. Clinicopathological analysis of idiopathic perforation of the gallbladder. Surg Today 2007; 37: $633-637$

3 Rapetti R, Scaglia E, Fangazio S et al. Pancreatobiliary reflux resulting in pancreatic ascites and choleperitoneum after gallbladder perforation. Case Rep Gastroenterol 2008; 2: 433-438

4 Kamisawa T, Okamoto A. Biliopancreatic and pancreatobiliary refluxes in cases with and without pancreaticobiliary maljunction: diagnosis and clinical implications. Digestion 2006; 73: 228-236

5 Menakuru SR, Kaman L, Behera A et al. Current management of gall bladder perforations. ANZ J Surg 2004; 74: 843-846

6 Beltran MA, VrackoJ, Cumsille MA et al. Occult pancreaticobiliary reflux in gallbladder cancer and benign gallbladder diseases. J Surg Oncol 2007; 96: 26-31

7 Itokawa F, Itoi T, Nakamura K et al. Assessment of occult pancreatobiliary reflux in patients with pancreaticobiliary disease by ERCP. J Gastroenterol 2004; 39: 988-994

8 Inagaki M1, Goto J, Suzuki S et al. Gallbladder carcinoma associated with occult pancreatobiliary reflux in the absence of pancreaticobiliary maljunction. J Hepatobiliary Pancreat Surg 2007; 14: 529-533

9 Horaguchi J, Fujita N, Kamisawa T et al. Pancreatobiliary reflux in individuals with a normal pancreaticobiliary junction: a prospective multicenter study. J Gastroenterol 2014; 49: 875-881 